\title{
Molecular disruption of RAD50 sensitizes human tumor cells to cisplatin-based chemotherapy
}

\author{
Waleed M. Abuzeid, ${ }^{1}$ Xiaoling Jiang, ${ }^{1}$ Guoli Shi, ${ }^{2}$ Hui Wang, ${ }^{1}$ David Paulson, ${ }^{1}$ Koji Araki, ${ }^{1}$ \\ David Jungreis, ${ }^{1}$ James Carney, ${ }^{3}$ Bert W. O'Malley Jr., ${ }^{1}$ and Daqing Li1 \\ 1Department of Otorhinolaryngology — Head and Neck Surgery, University of Pennsylvania School of Medicine, \\ Philadelphia, Pennsylvania, USA. 2Department of Otolaryngology — Head and Neck Surgery, and \\ ${ }^{3}$ Department of Radiation Oncology, University of Maryland School of Medicine, Baltimore, Maryland, USA.
}

\begin{abstract}
Platinum-based drugs that induce DNA damage are commonly used first-line chemotherapy agents for testicular, bladder, head and neck, lung, esophageal, stomach, and ovarian cancers. The inherent resistance of tumors to DNA damage often limits the therapeutic efficacy of these agents, such as cisplatin. An enhanced DNA repair and telomere maintenance response by the Mre11/Rad50/Nbs1 (MRN) complex is critical in driving this chemoresistance. We hypothesized therefore that the targeted impairment of native cellular MRN function could sensitize tumor cells to cisplatin. To test this, we designed what we believe to be a novel dominantnegative adenoviral vector containing a mutant $R A D 50$ gene that significantly downregulated MRN expression and markedly disrupted MRN function in human squamous cell carcinoma cells. A combination of cisplatin and mutant RAD50 therapy produced significant tumor cytotoxicity in vitro, with a corresponding increase in DNA damage and telomere shortening. In cisplatin-resistant human squamous cell cancer xenografts in nude mice, this combination therapy caused dramatic tumor regression with increased apoptosis. Our findings suggest the use of targeted RAD50 disruption as what we believe to be a novel chemosensitizing approach for cancer therapy in the context of chemoresistance. This strategy is potentially applicable to several types of malignant tumors that demonstrate chemoresistance and may positively impact the treatment of these patients.
\end{abstract}

\section{Introduction}

Platinum-based agents are the mainstay of chemotherapy for several tumor types, including ovarian, bladder, lung, and cancers of the head and neck (1). Cisplatin cytotoxicity results from the formation of DNA monoadducts and crosslinks, which, in turn, promote the development of apoptosis-inducing double-strand breaks (DSBs) during replication $(2,3)$. Unfortunately, cisplatin monotherapy often produces only a modest clinical tumor response (4-6). This has been attributed to tumor resistance against platinum-based treatment that is acquired after repeated cycles of therapy or due to an inherent resistance to these drugs (1).

Development of resistance to cisplatin is considered a major factor in disease relapse. Indeed, persistent locoregional disease and recurrence remain the main obstacles to improving prognosis (6). Even small increases in resistance are clinically relevant as the consequent dose escalations produce multiorgan toxicity (2). A major factor in the development of chemoresistance is the enhanced DNA repair ability of tumor cells preventing the accumulation of lethal DNA damage from cytotoxic agents (3, 7-9). Interruption of cellular DNA repair mechanisms can, therefore, sensitize tumor cells to DNA-damaging agents $(10,11)$.

In humans, DNA DSB repair involves the pathways of homologous recombination and nonhomologous end joining (12). The Mre11/Rad50/Nbs1 (MRN) complex is a critical component

Conflict of interest: The authors have declared that no conflict of interest exists.

Nonstandard abbreviations used: DSB, double-strand break; MRN, Mre11/Rad50/ Nbs1; MTM, mean tail moment; NCI, National Cancer Institute; SCC, squamous cell carcinoma.

Citation for this article: J. Clin. Invest. 119:1974-1985 (2009). doi:10.1172/JCI33816. in both of these pathways (13-15). Rad50 is an ATP-modulated DNA crosslinker, consisting of several structural features that are known to be essential to DNA repair activity. Three of the most important are the bipartite $\mathrm{ABC}$ domain, the Mre11 interaction sites, and the zinc hook region.

Like the related structural maintenance of chromosomes proteins, Rad50 is comprised of a coiled-coil sequence interposed between the 2 parts of the ABC ATPase domain. The N-terminal $\mathrm{ABC}$ harbors the Walker A motif, while the C-terminal ABC contains the Walker B motif and a highly conserved loop termed the signature motif (16). These sites have been shown to have both ATPase and adenylate kinase activity that are essential for all known functions of the MRN complex $(17,18)$. Binding of ATP to the Walker domains induces a conformational switch in the Rad50 protein that engages the $2 \mathrm{ABC}$ domains, forming a positively charged groove at the dimerization interface and increasing the affinity of this DNA-binding site for its substrate $(19,20)$. In essence, ATP governs the DNA binding of Rad50 through conformational switching and by cycling the binding affinity of the Rad50 ABC domain dimer $(19,21)$. Impairment of Rad50 adenylate kinase or ATPase activity through mutations in the signature motif or Walker domains leads to a Rad50-null phenotype and completely abrogates all ATP-dependent activities of the MRN complex. These include DNA binding, DNA tethering, subsequent unwinding of the DNA helix, and the resulting downstream activation of key effectors of DNA DSB repair $(17,22,23)$.

In all organisms studied thus far, Mre11 and Rad50 join to form a highly stable complex. The Mre11 interaction site on the Rad50 protein has been consistently confined to a region consisting of approximately 40 amino acids adjacent to the Rad50 ABC domain 
$(20,21)$. The hydrophobic region corresponding to this interaction site lies close to the Rad50 DNA-binding site, allowing Rad50 to form a coupled DNA-binding surface with Mre11 (21). The conformational changes in the Rad50 protein that occur upon ATP binding unwind and reposition DNA ends into the Mre11 active site and, hence, provide a mechanism whereby Rad50 regulates Mre11 exonuclease activity $(19,21)$. The efficiency of Mre11 nucleolytic cleavage is dependent upon the integrity of the Mre11 interaction site on the Rad50 protein.

The Rad50 ABC regions are at opposing ends of a long heptad repeat sequence that folds to form an antiparallel coiled-coil domain. The center of the Rad50 coiled-coil sequence harbors a conserved Cys-X-X-Cys-binding (CXXC-binding) motif within a region termed the zinc hook. The zinc hook is critical for zinc-mediated dimerization between Rad50 molecules and the resulting tethering link between sister chromatids in homologous recombination and DNA ends in nonhomologous end joining $(16,24,25)$. Coiled-coil polypeptides, consisting of only the CXXC motif, are capable of dimerizing with each other (26). The CXXC motif is located at the apex of the Rad50 coiled-coil and forms a tethering link between the coiled-coils of 2 different, oppositely protruding MRN complexes (12). Each MRN complex is a functional unit, consisting of a heterotetramer with Mre $11_{2} /$ $\operatorname{Rad} 50_{2}$ stoichiometry. Each $\mathrm{Mre} 11_{2} / \operatorname{Rad} 50_{2}$ heterotetramer binds to an opposing DNA end or sister chromatid and is linked to the other by dimerization of the Rad50 hook domains (26). Loss of the Rad50 hook structural domain abrogates the DNA repair functions of MRN, producing spontaneous chromosomal instability and hypersensitivity to chemotherapy and radiation $(24,27)$. Thus, the integrity of the Rad50 ATPase and zinc hook regions as well as the Mre11 interaction site is critical to the process of DNA DSB repair by the MRN complex.

Rad50 also has a role in telomere maintenance (28). MRN generates the $3^{\prime}$ overhangs at the ends of chromosomes that act as replication primers for telomerase, a reverse transcriptase expressed by most cancers that counteracts the loss of telomeric DNA (15, 29). These actions depend upon the molecular bridging of DNA strands via the Rad50 hook domain as well as intact adenylate kinase function via the $\operatorname{Rad} 50$ signature motif $(17,24)$. Disruption of Rad50 function produces severe telomere shortening and cell death in transformed and tumor cells (24). The MRN complex is also a key component of telomere protection mechanisms. Indeed, Rad50 is physically associated with key shelterins. These are proteins that cap telomeres and prevent their recognition as DSBs and subsequent inappropriate processing by DNA repair pathways, but which also prevent telomerase from access to the ends of telomeres $(30,31)$. $\operatorname{Rad} 50$ is known to downregulate the telomeric association of key shelterins, promoting increased telomerase activity and telomere lengthening. Disruption of Rad50 function prevents MRN-mediated phosphorylation of shelterins and their resulting dissociation from telomeres. Consequently, telomere ends cannot be lengthened by telomerase and telomeric shortening ensues (30).

The critical role of Rad50 in DNA repair and telomere maintenance served as the key rationale for our development of a Rad50targeted dominant-negative adenoviral vector that can disrupt MRN function and, thereby, sensitize squamous cell carcinoma (SCC) to cisplatin-based chemotherapy. The present study is the first to our knowledge to translate the knowledge of Rad50 structure and function into a potential clinically applicable cancer treatment.

\section{Results}

SCC tumor cells natively express wild-type MRE11, RAD50, and NBS1 proteins. The MRN complex is highly conserved, but mutations in Nbs1, Mre11, and Rad50 genes, which produce chromosomal instability syndromes and an inherent susceptibility to DNA-damaging agents, have been identified (32-34). To avoid this potential confounding factor, we confirmed expression of wild-type MRE11 (Supplemental Figure 1; supplemental material available online with this article; doi:10.1172/JCI33816DS1), RAD50 (Supplemental Figure 2), and NBS1 (Supplemental Figure 3) proteins in JHU012 and JHU029 human SCC cells, which are derived from cancers of the head and neck region. All lanes from both SCC cell lines demonstrated the presence of wild-type MRE11 (81 kDa), RAD50 (153 kDa), NBS1 (95 kDa), and $\beta$-actin (control) proteins (42 kDa) (Figure 1). Confirmation of wild-type expression of the p53 gene and screening of the genetic background of the JHU cell lines was performed and has been reported previously (35-38). The JHU012 and JHU029 cells, derived as clones from parental cell lines, were used for all experiments.

Adenoviral vector containing mutant RAD50 gene induces transgene expression of mutant RAD50 protein in tumor cells. We designed what we believe to be a novel adenoviral vector consisting of a recombinant serotype 5 adenovirus, which we termed Ad-RAD50. This vector contains a gene sequence encoding a fragment of the RAD50 zinc hook region, with an intact CXXC motif, but completely missing the regions that encode the ATPase regions and the adjacent MRE11 interaction sites (Figure 2A). The resulting mutant RAD50 protein is not able to bind to either ATP or MRE11 due to the absence of these regions. This abrogates MRN-mediated DNA DSB repair and telomere maintenance as well as MRN complex formation, respectively. Expression of the 13-kDa mutant RAD50 protein was detected by Western blot in JHU012 tumor cells infected with Ad-RAD50 (Figure 2B and Supplemental Figure 4).

The trans-interaction of the mutant RAD50 book domain with endogenous RAD50 protein through zinc-mediated dimerization interrupts and downregulates endogenous $M R N$ expression. Trans-interaction of the mutant RAD50 hook domain with endogenous, wild-type RAD50 protein via zinc-mediated dimerization resulted in the formation of a single MRE $11_{2} /$ RAD $50_{2}$ heterotetramer bound to mutant RAD50. Absence of the MRE11 interaction site on the mutant RAD50 component prevented assembly of a second, functional MRE11 $2 / \mathrm{RAD} 0_{2}$ heterotetramer on the opposing DNA strand or sister chromatid. Impairment of MRE11 and RAD50 interaction had a subsequent effect on NBS1, as this protein can only interact with the MRE11 $12 /$ RAD50 $0_{2}$ heterotetramer via binding sites on MRE11. These binding interactions were demonstrated through a series of coimmunoprecipitation experiments. JHU012 head and neck cancer cells were divided into the following groups: untreated controls, treated with cisplatin, infected with Ad-RAD50, or treated with both cisplatin and Ad-RAD50 (Figure $3 A)$. Zinc chloride was added to selected groups prior to coimmunoprecipitation as zinc is known to be essential for normal cellular function and is a requirement for the dimerization interaction between the hook regions of adjacent RAD50 proteins $(12,24)$. In all groups, anti-human MRE11 antibody was able to coimmunoprecipitate the wild-type RAD50 protein, confirming the binding interaction between MRE11 and wild-type RAD50 proteins (21). In cells treated with Ad-RAD50, either alone or in combination with cisplatin, anti-MRE11 antibody was able to additionally pull down the mutant RAD50 protein but only in the presence of zinc 

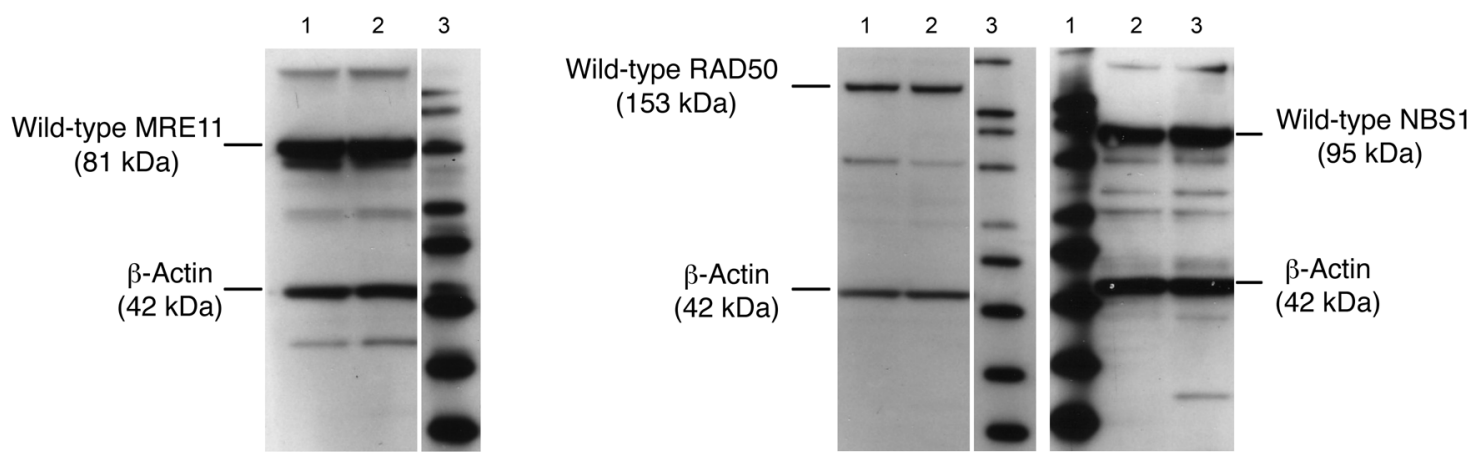

\section{Figure 1}

Native MRN expression in head and neck SCC cells. Native expression of MRE11, RAD50, and NBS1 was confirmed in both JHU012 and JHU029 SCC cells by Western blot. Lane 1, JHU029 cells; lane 2, JHU012 cells; lane 3, MagicMark Western Protein Standard with markers of $20,30,40,50,60,80,100,120$, and $220 \mathrm{kDa}$ from bottom to top. Lanes were run on the same gel but were noncontiguous as indicated by the vertical white lines.

chloride (Figure 3A). The physical interactions of MRE11, wildtype RAD50, and mutant RAD50 following Ad-RAD50 infection were further elucidated through a series of confocal microscopy experiments. JHU012 cells were left untreated, treated with cisplatin, or infected with Ad-RAD50 with or without cisplatin. Cells were treated with anti-human RAD50 antibodies targeted against the N-terminal of the wild-type protein and anti-human RAD50 (zinc hook region) antibodies that label the mutant RAD50 protein or anti-human MRE11 antibodies. Secondary antibodies were used to label the respective proteins for visualization under laser scanning confocal microscopy. All groups demonstrated colocalization of the signals representing wild-type RAD50 and MRE11, suggesting a direct interaction between these proteins (Figure $3 \mathrm{~B}$ and Supplemental Figures 5-7). Only cells that had been infected with Ad-RAD50 demonstrated a signal corresponding to the mutant RAD50 protein. In infected cells, there was marked overlap of the nuclear signals corresponding to mutant RAD50 and wildtype RAD50, indicating a direct interaction between these proteins (Figure 3C and Supplemental Figures 8-10).

These results highlighted 2 key findings: first, that MRE11, wild-type RAD50, and mutant RAD50 existed as a complex and, second, that the mutant RAD50 component bound with the complex via a zinc-dependent interaction of its hook region with the complimentary hook region of wild-type RAD50. The wildtype RAD50, in turn, interacted with MRE11 via its intact interaction site. The zinc-dependent nature of the mutant RAD50 interaction with the MRN complex is evidence against direct interaction between mutant RAD50 and MRE11, as supported by the absence of the MRE11 interaction site coding region in the gene construct. As shown in Figure 3D, a significant downregulation of wild-type MRE11 $(P<0.005)$ (Supplemental Figure 11), RAD50 $(P<0.001)$ (Supplemental Figure 12), and NBS1 $(P<0.04)$ (Supplemental Figure 13) was detected in JHU012 tumor cells infected with Ad-RAD50. Our data indicated that the ability of the mutant RAD50 protein to interact with the wildtype $\mathrm{MRE} 11_{2} / \mathrm{RAD} 50_{2}$ heterotetramer induces a dominant-negative downregulation of the MRN complex, since a second heterotetramer cannot form around the mutant protein and therefore results in disruption of the MRN complex.

Mutant RAD50 transgene expression enhances the cytotoxicity of cisplatin in vitro. Cell growth was measured in JHU012 and JHU029 cell lines using the 3-(4,5-dimethylthiazol-2-yl)-2,5-diphenyltet- razolium bromide (MTT) cell growth assay (Figure 4). Cells were subjected to 6 interventions: no treatment controls, treated with DL312 empty control virus, treated with Ad-RAD50, treated with cisplatin, treated with DL312 and cisplatin, and treated with Ad-RAD50 and cisplatin. Adenoviral vectors were introduced at a MOI of 10. Cisplatin was instilled at a concentration of $0.3 \mu \mathrm{g} / \mathrm{ml}$ and $0.4 \mu \mathrm{g} / \mathrm{ml}$ for JHU012 and JHU029 cell lines, respectively. This cisplatin concentration represented the $\mathrm{IC}_{50}$ dose for each cell line. Control and DL312-treated SCC cells exhibited persistent logarithmic growth. Cisplatin, alone or with DL312 control virus, produced a reduction in cell growth relative to controls in both cell lines $(P<0.01)$. Ad-RAD50 monotherapy also produced a significant reduction in cell proliferation, without the need for an external DNA-damaging agent $(P<0.01)$. Combination therapy with Ad-RAD50 and cisplatin persistently suppressed SCC cell growth within 48 hours of treatment initiation, and this cytotoxic effect was significant compared with either treatment alone $(P<0.01)$. On day 6 , Ad-RAD50 and cisplatin combination therapy produced a $67.8 \%, 53.2 \%$, and $33.6 \%$ reduction in JHU012 cell density and a $55.4 \%, 35.2 \%$, and $33.3 \%$ decrease in JHU029 cell number relative to controls, cisplatin monotherapy, and Ad-RAD50, respectively. Mutant RAD50 transgene expression was, therefore, capable of potentiating the cytotoxic effect of cisplatin in SCC.

Enhanced cytotoxicity is associated with increased DNA DSB damage. A key mechanism of enhanced cytotoxicity observed in combination Ad-RAD50 and cisplatin therapy was elucidated by neutral comet assay. This technique allowed for the quantification of DNA DSBs in individual cells treated with DNA-damaging agents. Undamaged, high-molecular-weight DNA does not migrate under an electrophoretic field, producing the comet "head." DNA damage results in relaxation of supercoils and migration of the broken ends toward the anode, producing the comet "tail" (39) (Figure $5 \mathrm{~A})$. The tail moment, which correlates with the amount of DNA damage, is a function of the length and intensity of the comet tail. Tail moment was averaged across 30-70 cells within each group to derive the mean tail moment (MTM) for the JHU012 (Figure 5B) and JHU029 (Figure 5C) cell lines.

JHU012 and JHU029 SCC cells were assessed for DSB damage. A MOI of 10 was used for adenoviral infection. The amount of DNA damage measured across both cell lines correlated with the cytotoxic effects observed for each group in the respective cell proliferation assays. Cells treated with cisplatin $(0.3 \mu \mathrm{g} / \mathrm{ml}$ 


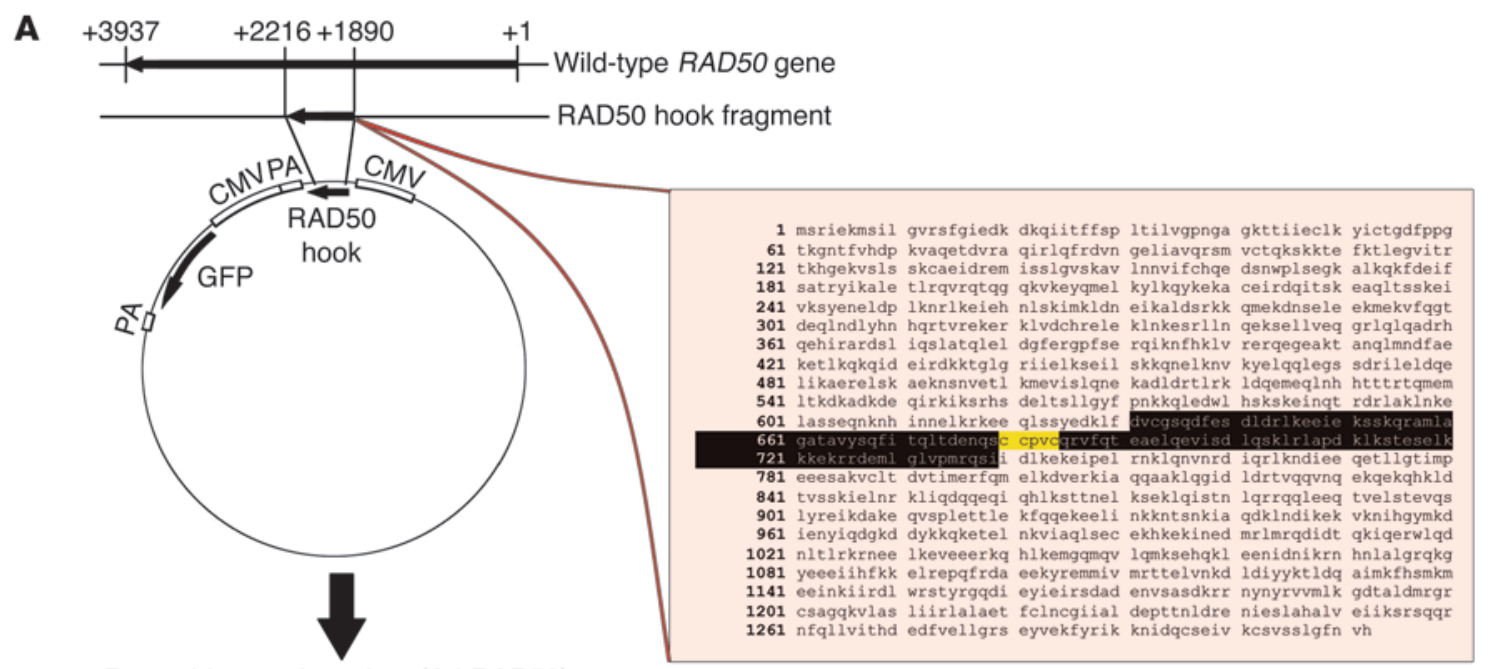

Recombinant adenovirus (Ad-RAD50)

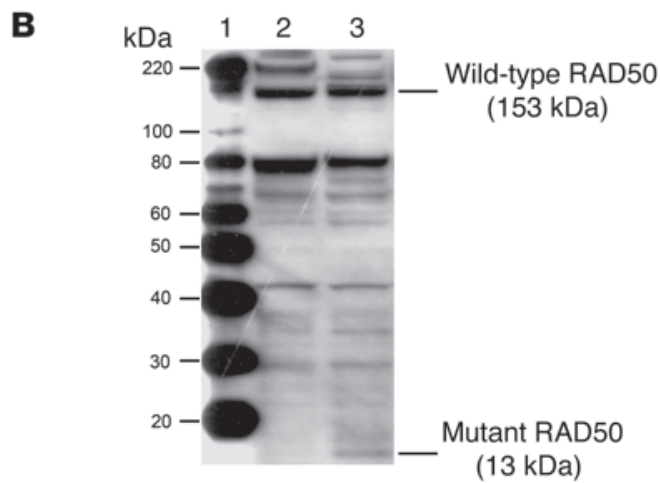

\section{Figure 2}

Construction and function of the Ad-RAD50 vector. (A) A 326-base pair mutant RAD50 hook fragment from the wild-type RAD50 gene was cloned into a recombinant adenovirus vector to construct Ad-RAD50. The amino acid sequence of the integrated construct (residues 631-739) is highlighted in black, with the zinc hook region marked in yellow. PA, polyadenylation site. (B) Both the wild-type RAD50 protein and the mutant RAD50 protein were detected by Western blot in JHU012 tumor cells that were infected with Ad-RAD50 adenovirus. Lane 1, MagicMark Western Protein Standard; lane 2, JHU012 cells with no treatment; lane 3, JHU012 cells infected with Ad-RAD50.

for JHU012; $0.4 \mu \mathrm{g} / \mathrm{ml}$ for JHU029) exhibited an increased MTM relative to control cells (JHU012, 793 vs. 188, $P<0.001$; JHU029, $1,385$ vs. $365, P<0.001)$. DL312 control virus induced a small increase in MTM versus controls, but this is attributable to the reported increased in apoptosis following DL312 infection of cancer cells (40). In the JHU012 cell line, Ad-RAD50 monotherapy was also able to produce a significantly greater MTM than the control, DL312, cisplatin, or DL312 in combination with cisplatin groups (3136 vs. 188, 563, 793, 1,561, respectively; $P<0.001)$. In the JHU029 cell line, Ad-RAD50 monotherapy significantly increased MTM relative to control and DL312 groups (867 vs. 365 and 425, respectively; $P<0.001$ ) but did not induce as much DSB damage as cisplatin or cisplatin combined with DL312 treatment (867 vs. 1,385 and 1,356 , respectively; $P<0.002$ ). Considering that cellular DNA routinely sustains about $10^{4}$ DNA damage events per cell per day and that a single DSB can, if unrepaired, induce cell death, the observed increase in DSBs and enhanced cytotoxicity following Ad-RAD50 infection may be secondary to the persistence of naturally occurring DNA damage in the face of impaired MRN DNA repair function $(32,41)$.
Combining Ad-RAD50 and cisplatin produced a greater MTM (JHU012, 4,258; JHU029, 1,999) than nontreatment and treatment with DL312 controls $(P<0.001$ for JHU012 and JHU029), cisplatin alone or in combination with DL312 $(P<0.001$ for JHU012; $P<0.003$ for JHU029), and Ad-RAD50 monotherapy $(P<0.02$ for JHU012; $P<0.001$ for JHU029). This represents an increase in DNA DSBs of $2,171 \%, 656 \%, 437 \%, 173 \%$, and $36 \%$ versus nontreatment and treatment with DL312 controls, cisplatin, DL312 and cisplatin, and Ad-RAD50 alone, respectively, in JHU012 cells. In JHU029 cells, combined treatment with Ad-RAD50 and cisplatin increased DNA DSBs by $448 \%, 371 \%, 131 \%, 44 \%$, and $47 \%$ relative to nontreatment and treatment with DL312 controls, Ad-RAD50 alone, cisplatin alone, and cisplatin with DL312, respectively. The potent DNAdamaging effect associated with combination Ad-RAD50 and cisplatin correlates with the observed cytotoxicity of this treatment.

Enhanced cytotoxicity is associated with telomere shortening. RAD50 plays an important role in the maintenance of telomeres $(24,29$, 42). Telomere PNA FISH was used to analyze the length of cell telomeres from each group in JHU012 SCC cells. Telomere staining intensity (Figure 6A) is linearly proportional to telomere 
A

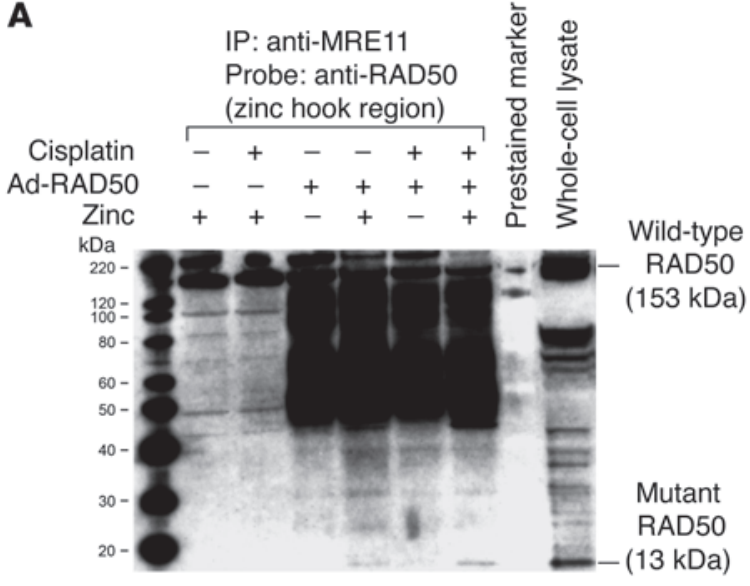

D Wild-type MRE11

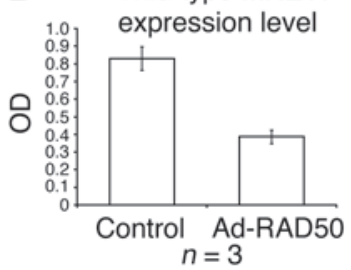

Wild-type RAD50

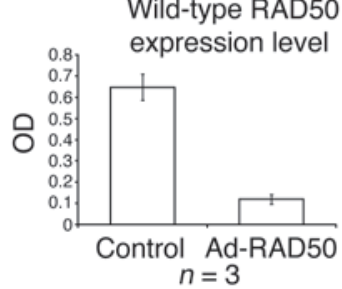

B

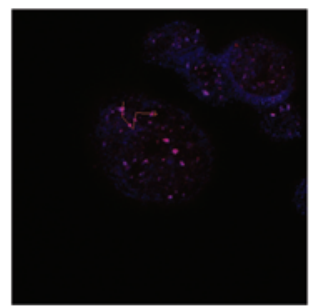

Profile
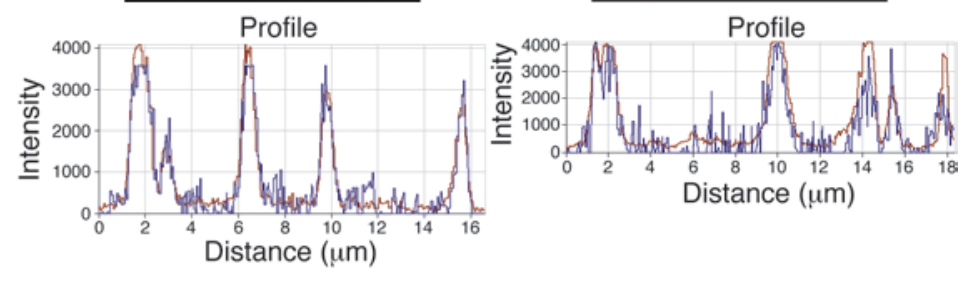

C

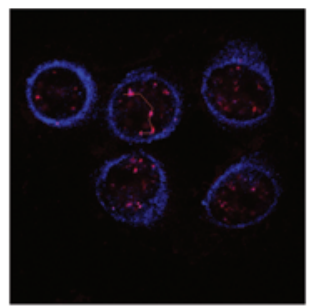

Profile

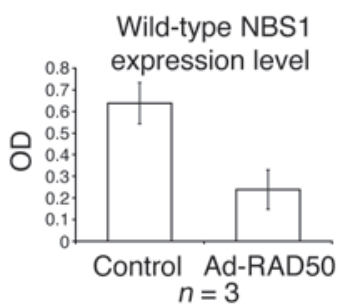

Figure 3

Mutant RAD50 protein interacts with endogenous RAD50 and downregulates the MRN complex. (A) Anti-MRE11 antibody coimmunoprecipitated wild-type RAD50 in all cells, suggesting a direct interaction between these proteins. Mutant RAD50 was only coimmunoprecipitated in infected cells treated with zinc, indicating that the interaction between mutant RAD50 and the wild-type MRN complex is mediated by zinc-dependent dimerization of RAD50 hook domains and not direct interactions between mutant RAD50 and MRE11. All lanes shown were run simultaneously on a single gel as contiguous lanes. (B) Multiple MRN nuclear foci are seen at sites of DNA damage in JHU012 cells treated with Ad-RAD50 and cisplatin. Original magnification, $\times 63$. There is spatial overlap of the signals corresponding to wild-type RAD50 (blue) and wild-type MRE11 (red), highly suggestive of a binding interaction between these proteins. (C) Nuclear foci from JHU012 cells treated with Ad-RAD50 and cisplatin (original magnification, $\times 63$ ) demonstrate spatial overlap of the wild-type (red) and mutant RAD50 (blue) proteins, further suggesting direct binding between these proteins. (D) Downregulation of wild-type MRE11 $(P<0.005)$, RAD50 $(P<0.001)$, and NBS1 proteins $(P<0.04)$ is seen after infection of JHU012 cells with Ad-RAD50 relative to noninfected controls. (Mean optical density of Western blot bands \pm SEM is shown.) This is attributable to the demonstrated dimerization between mutant and wild-type RAD50 and inability of the mutant protein to directly bind MRE11. Consequently, only a single MRE $11_{2} / \mathrm{RAD} 50_{2}$ heterotetramer can form around the wild-type RAD50 with no complimentary heterotetramer assembling around mutant RAD50.

length. Quantification of telomere staining (Figure 6B) indicated that nontreated control JHU012 cells had the highest staining intensity of 759.0 and, thus, the longest telomeres. Cisplatin is known to preferentially bind to runs of 2 or more guanine residues. Consequently, the (TTAGGG) human telomeric sequence forms an ideal target for cisplatin. Indeed, cisplatin is known to induce telomere shortening and apoptosis in human cells (43). In JHU012 cells, cisplatin $(0.3 \mu \mathrm{g} / \mathrm{ml})$ caused a significant reduction in telomere staining intensity relative to controls (248.8 vs. 759.0; $P<0.01$ ). Ad-RAD50 monotherapy (MOI 10), through an effect on $\mathrm{MRN}$-mediated telomere maintenance as well as direct DNA damage, also produced a marked reduction in telomere staining intensity to 187.0. Combination therapy with Ad-RAD50 (MOI 10) and cisplatin $(0.3 \mu \mathrm{g} / \mathrm{ml})$ reduced telomere staining intensity to 65.0 , indicating that telomeres were shorter than in all other groups $(P<0.01)$. Therefore, impairment of RAD50 telomere maintenance and DNA repair function can facilitate cisplatin-mediated telomere shortening. This effect is likely to have contributed to the observed cytotoxic effect of combination treatment, since telomere shortening beyond a critical threshold induces cell death.
RAD50 disruption enhances the outcome of cisplatin-based chemotherapy in human head and neck SCC xenografts. Human head and neck SCC xenografts were established in nude mice and were used to further evaluate whether the in vitro cytotoxicity caused by the disruption of normal MRN function translates into a beneficial effect on in vivo tumor growth. JHU012 SCC cells were injected subcutaneously into the dorsal flanks of 60 nude mice at day 0 . Treatments were administered on day 13 , and the animals were sacrificed on day 24. Tumor volumes were measured externally throughout the duration of the experiment (Figure 7A). For increased accuracy in the determination of treatment efficacy, tumors were surgically exposed by raising skin flaps and volumes were measured internally (as described in the Methods), immediately before the initiation of treatment on day 13 and again at the conclusion of the experiment on day 24 (Figure 7B).

There was no significant difference in tumor size prior to initiating treatment in any of the groups. Control group tumors exhibited sustained growth following subcutaneous cancer cell injection (Figure 7A) and, between days 13 and 24, showed a 186\% increase in tumor volume relative to pretreatment size. Cisplatin mono- 
A

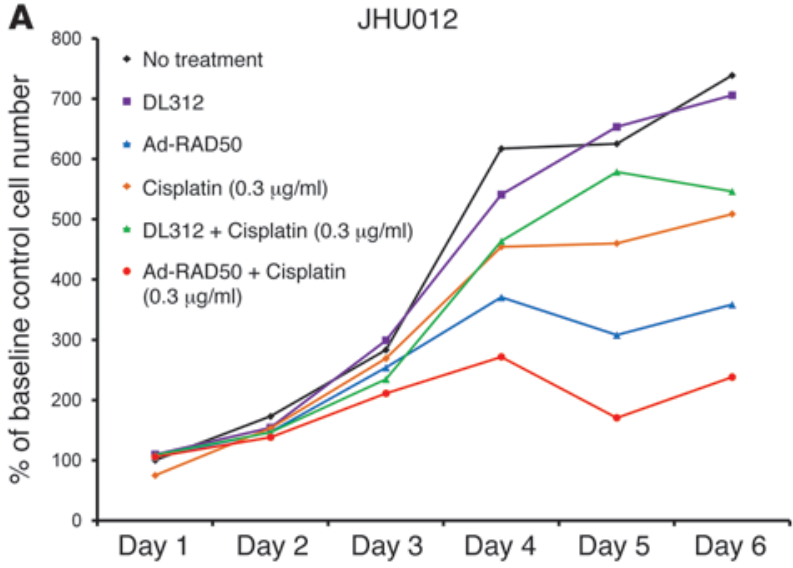

B

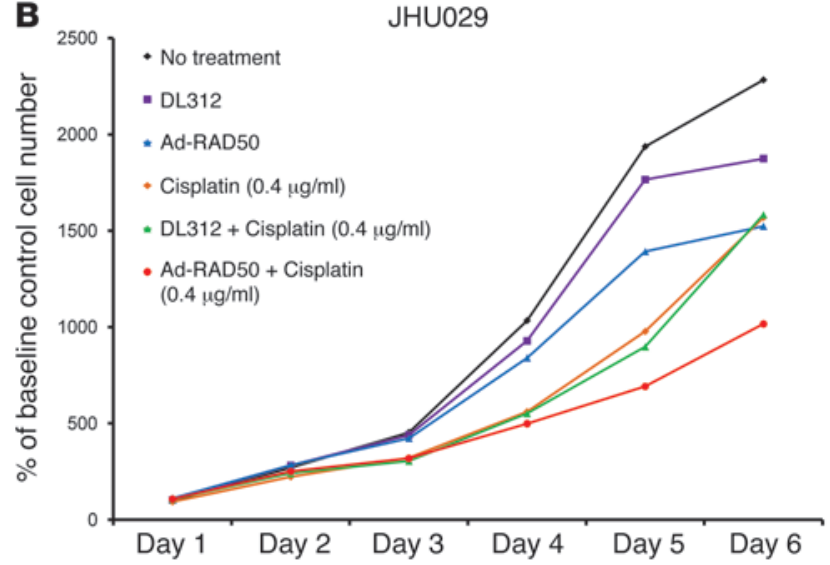

Figure 4

Ad-RAD50 enhances cisplatin-induced suppression of SCC cell growth. (A) Combining Ad-RAD50 and cisplatin in JHU012 SCC cells produced a reduction in cell growth within 48 hours and a persistent suppression of cell proliferation relative to all other groups $(P<0.01)$. DL312 indicates DL312 control virus. (B) The addition of Ad-RAD50 to cisplatin treatment in JHU029 cells produced the greatest reduction in cell growth versus all other groups $(P<0.01)$. Cells were cloned from parental cell lines. Mean optical density $\pm \mathrm{SEM}$ is shown.

therapy, given as a single $3 \mathrm{mg} / \mathrm{kg}$ intraperitoneal dose, suppressed tumor growth (Figure 7A), with tumors showing a $71 \%$ increase in internally measured tumor volume compared with pretreatment levels (Figure 7B). This growth reduction was significant versus controls (percentage of change in mean tumor volume for treatment group divided by change in mean tumor volume in control group $[\% T / C]=60 \% ; P<0.03$ ) (Figure $7 \mathrm{C}$ ). Increasing the cisplatin dose to $5 \mathrm{mg} / \mathrm{kg}$ significantly enhanced tumor growth suppression relative to controls (Figure 7A), with internal tumor volume increasing by $40 \%$ relative to pretreatment levels $(\% T / C=49 \% ; P<0.003$ ) (Figure $7, \mathrm{~B}$ and C). According to National Cancer Institute (NCI) standard criteria, this value indicates that cisplatin has no appreciable efficacy against JHU012, suggesting that this human head and neck SCC has inherent chemoresistance to cisplatin (44).

A single intratumoral injection of Ad-RAD50 at day 13 induced a sustained and significant suppression of tumor growth relative to controls (Figure 7). Internally measured tumor dimensions indicated that volume increased by $39 \%$ between day 13 and day $24(\% \mathrm{~T} / \mathrm{C}=48 \% ; P<0.02)$ (Figure $7, \mathrm{~B}$ and $\mathrm{C})$. Combined therapy with intratumoral Ad-RAD50 and intraperitoneal cisplatin $(3 \mathrm{mg} / \mathrm{kg}$ ) produced a marked tumor suppressive effect (Figure $7 \mathrm{~A})$. Determination of the tumor volume through internal measurement revealed that tumor volume increased by only $0.6 \%$ compared with pretreatment volume and that this combination therapy met NCI criteria for significant antitumor efficacy (\%T/C $=35 \% ; P<0.001$ vs. controls, $P<0.05$ vs. cisplatin $[3 \mathrm{mg} / \mathrm{kg}]$ monotherapy, $P<0.05$ vs. cisplatin $[5 \mathrm{mg} / \mathrm{kg}]$ with Ad-DL312, $P<0.05$ vs. Ad-RAD50 alone) (Figure 7, B and C). An even greater tumor suppressive effect was achieved by combining Ad-RAD50 with a higher dose of cisplatin $(5 \mathrm{mg} / \mathrm{kg}$ ) (Figure $7 \mathrm{~A})$. Tumor volume decreased by $24 \%$ relative to pretreatment levels $(\% T / C=27 \%$; $P<0.001$ vs. control, cisplatin $[3 \mathrm{mg} / \mathrm{kg}]$, cisplatin $[5 \mathrm{mg} / \mathrm{kg}]$ with DL312, and Ad-RAD50 alone) (Figure 7, B and C).

These results indicated that Ad-RAD50 is able to reverse the inherent cisplatin resistance of JHU012 SCC cells. This effect was secondary to transgene expression of the mutant RAD50 gene and not a consequence of adenovirus-mediated cytotoxicity, since the combination of cisplatin with the DL312 control virus was significantly less efficacious than combination cisplatin with Ad-RAD50. The improved antitumor efficacy of lower dose cisplatin with Ad-RAD50 versus higher dose cisplatin therapy alone could prove valuable in future human clinical trials of RAD50-disrupting therapies by permitting the use of lower and, thus, less toxic doses of cisplatin, while achieving an improved antitumor response. No mice exhibited weight loss after the initiation of treatment, and there was no significant difference in weight change between treatment groups and controls. No mice died during the experiment. This suggests mutant $R A D 50$ gene transfer, with or without chemotherapy, is well tolerated.

TUNEL staining was used on tumor frozen sections, and the percentage of apoptotic cells was determined using IPLab software (Figure 8, A and B). Nontreated controls had low levels of apoptosis per high-powered field (0.12\%). Cisplatin induced significantly more apoptosis, alone and combined with DL312 (1.26\% and 1.42\%, respectively; $P<0.001)$. Ad-RAD50 infection also enhanced apoptosis $(0.84 \% ; P<0.005)$. Combining Ad-RAD50 with cisplatin markedly increased the amount of apoptosis in mice subjected to both the $3 \mathrm{mg} / \mathrm{kg}$ and $5 \mathrm{mg} / \mathrm{kg}$ dose of cisplatin (3.46\% and $4.43 \%$, respectively) relative to all other groups $(P<0.001)$. Within the combination treatment groups, there was no significant difference in apoptosis between the low and higher doses of cisplatin, consistent with the similar tumor volume reduction observed in these groups.

\section{Discussion}

Cisplatin and other platinum-based drugs are commonly used as first-line treatment in many cancers. DNA DSBs are the first morphologic change associated with cell death in cisplatintreated tumors. Cisplatin exerts a dose-dependent effect and, at higher concentrations, overwhelms DNA repair mechanisms, killing a larger fraction of tumor cells (45). However, cancer cells can develop resistance through enhanced DNA damage repair (2). From a clinical viewpoint, increasing resistance necessitates increased cisplatin (or any chemotherapy agent) dosing and a subsequent risk for increased adverse effects and treatment- 
A Nontreatment controls
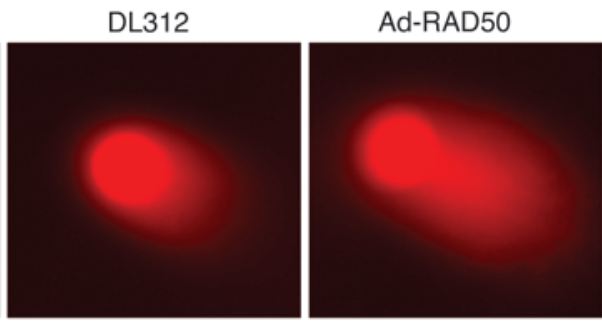

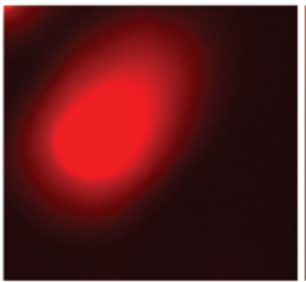

Cisplatin

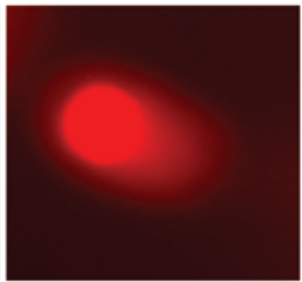

Cisplatin + DL312

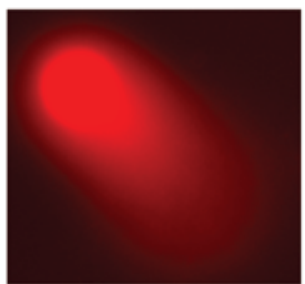

Cisplatin + Ad-RAD50

B

JHU012

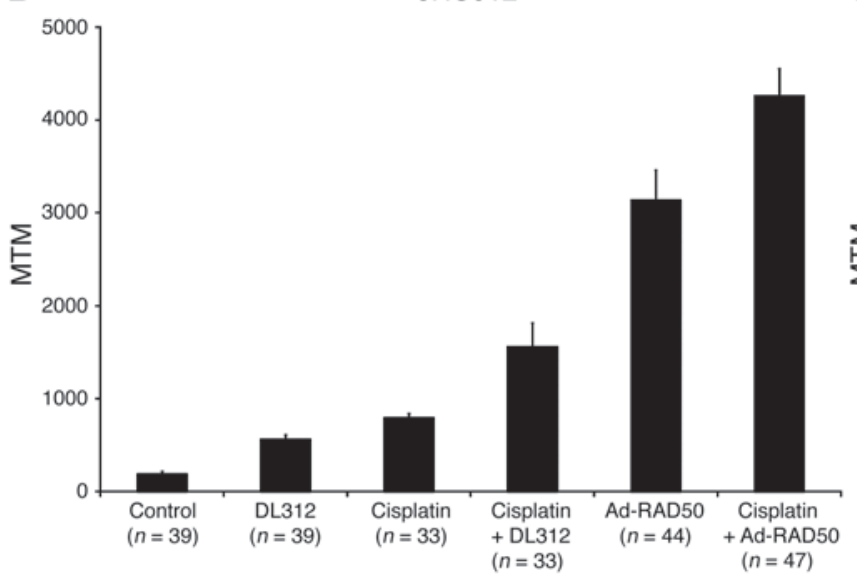

JHU029

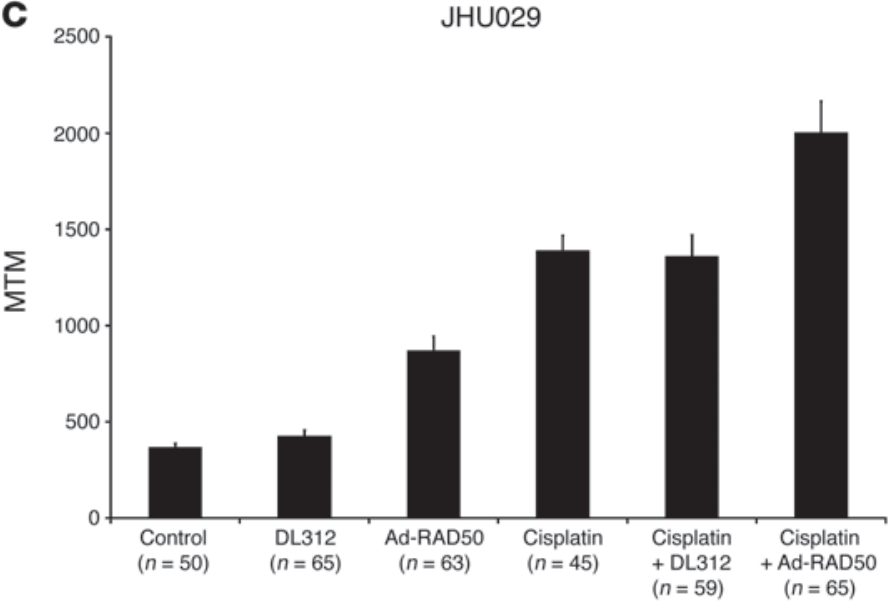

Figure 5

Ad-RAD50 enhances cisplatin-induced DNA damage. (A) Neutral comet assay was used to quantify the level of DNA DSB damage in JHU012 (shown) SCC cells. Damaged, fragmented DNA migrates toward the anode, producing a comet tail. Original magnification, $\times 20$. (B) MTM as measured in the JHU012 cell line. The MTM is a correlate of DNA DSB damage. MTM \pm SEM is shown. Ad-RAD50 combined with cisplatin increased the number of DNA DSBs by $437 \%$ relative to cisplatin monotherapy $(P<0.001)$ and by $173 \%$ compared with Ad-RAD50 alone $(P<0.02)$, suggesting that the enhanced cytotoxic effect of combined therapy is secondary to increased DNA DSB damage. (C) MTM as measured in the JHU029 cell line. MTM \pm SEM is shown. Ad-RAD50 combined with cisplatin significantly increased DSB damage compared with all other groups $(P<0.003)$.

limiting toxicities. Alternatively, increased resistance can lead to reduced efficacy of the chemotherapeutic agent for ongoing or subsequent treatment.

In the present study, a recombinant adenoviral vector harboring a mutant RAD50 construct was created. The resulting mutant RAD50 protein has a preserved hook domain but lacks the MRE11 interaction sites as well as the $\mathrm{N}$ - and C-terminal ATPase domains necessary for DNA repair function. Coimmunoprecipitation and confocal studies demonstrated that the mutant RAD50 retains the ability to dimerize in a zinc-dependent manner with wildtype RAD50 via its hook domain but is itself incapable of binding directly to MRE11. So although a functional MRE11 $2 / \mathrm{RAD} 50_{2}$ heterotetramer could form around wild-type RAD50, protein assembly could not coordinate around the mutant RAD50 protein, and a fully functional MRN octamer did not form. This induces a dominant-negative effect that disrupts the MRN complex, as evidenced by the significantly downregulated expression of MRE11, RAD50, and NBS1 proteins. This dominant-negative effect has also been previously demonstrated through the use of RNA interference. Both transient and stable knockdown of the wild-type Rad50 gene induced a concurrent suppression in Mre11 and Nbs1 protein levels $(27,46)$. The in vitro and in vivo studies described here demonstrate the biological effects resulting from the disruption of MRN function.

The MRN complex is essential to DSB detection, DSB repair, and telomere maintenance and in cell cycle checkpoint control $(16,29,42,47)$. The molecular disruption of the MRN complex through expression of mutant RAD50 sensitized tumor cells to cisplatin-based chemotherapy. Upon treatment with cisplatin, the SCC cells infected with Ad-RAD50 showed significantly more DSBs than produced by cisplatin therapy alone. Although previous studies have shown that mutations in the Rad50 ATPase region completely abrogate the DNA repair function of MRN and sensitize cells to DNA-damaging agents $(17,24,26)$, this is 
A Nontreatment controls

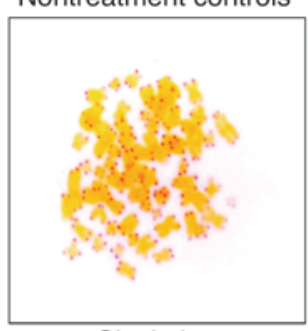

Cisplatin
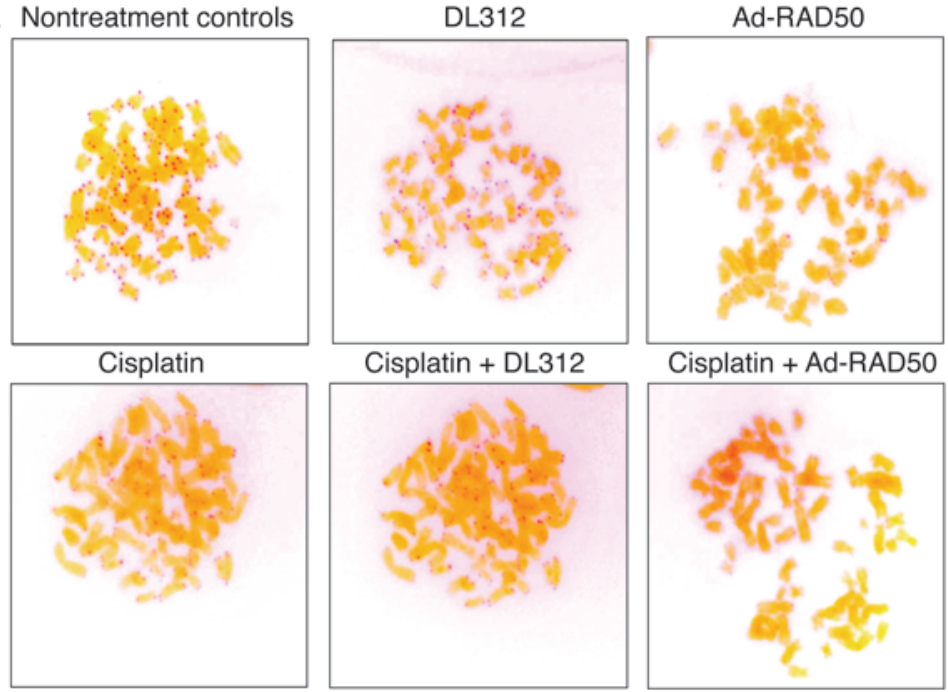

Cisplatin + Ad-RAD50
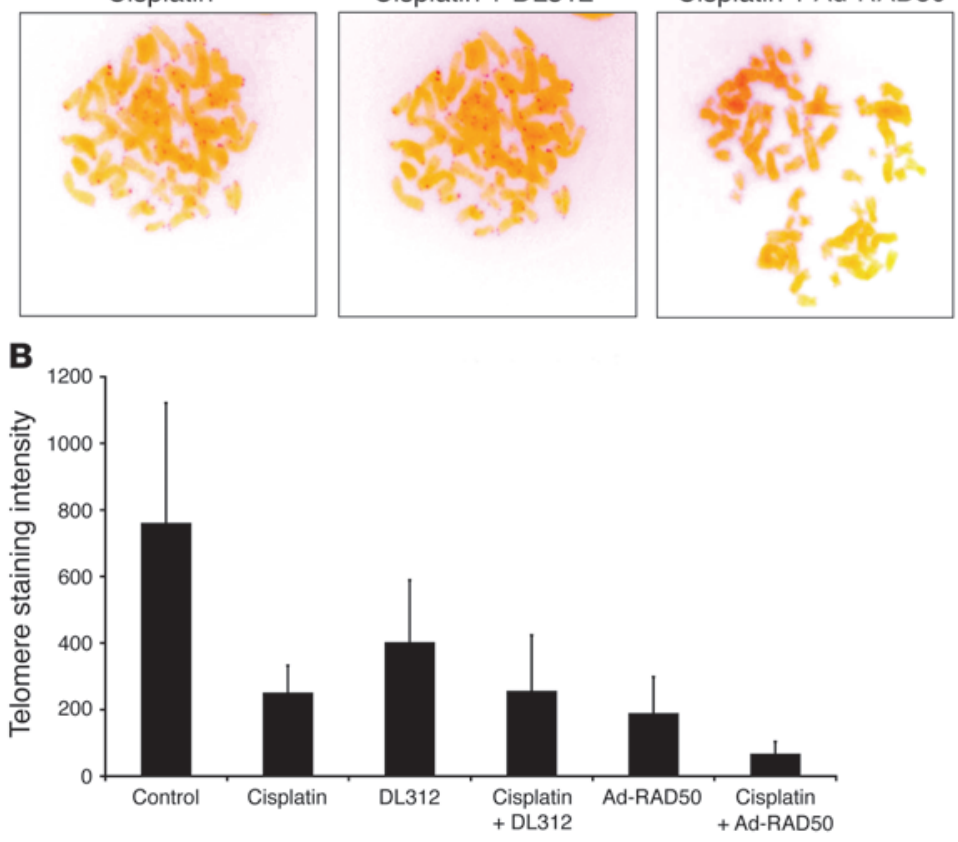

Figure 6

Ad-RAD50 and cisplatin combination treatment enhances telomere shortening in JHU012 SCC cells. (A) PNA FISH stains telomeres with a red signal, the intensity of which is proportional to telomere length. Original magnification, $\times 100$. (B) Software-based quantification of telomere staining intensity indicated that combining Ad-RAD50 with cisplatin markedly attenuated telomere length by $73.9 \%$ versus cisplatin alone and by $65.2 \%$ versus Ad-RAD50 $(P<0.01)$. Telomere shortening beyond a critical threshold induces apoptosis, contributing to the cytotoxic effect of combination therapy in tumor cells. Mean \pm SEM is shown.

the first report to our knowledge translating a RAD50-disrupting approach to antitumor therapy, shown here in vitro and in human head and neck SCC xenografts.

The chemosensitizing effect of Ad-RAD50 may also be attributed to disruption of MRN-mediated telomere maintenance. Activation of telomere maintenance mechanisms to compensate for loss of telomeric nucleotides during replication is critical for cellular immortalization (29). A key telomere maintenance mechanism that could potentially be affected by MRN disruption is telomerase, the activation of which is critical for immortality in a significant proportion of cancers (48). The ends of human chromosomes possess long G-overhangs that are generated by $\mathrm{MRN}$ and are used as replication primers for telomerase-mediated telomere extension (42). An intact Rad50 ATPase region is critical for telomere maintenance (17). The absence of ATPase domains in the mutant Rad50 construct disrupts MRN-mediated telomere maintenance. Without MRN, G-overhangs are not generated and telomerase function is impaired (29). A second telomere mainte- nance mechanism, termed alternative lengthening of telomeres (ALT), is often used by immortal cells (46). In contrast to the progressive, replication-dependent attrition of telomeres seen in telomerase-inactivated cells, deactivation of ALT can induce much more rapid and profound telomere degradation, termed telomere rapid deletion $(49,50)$. In cells that are known to use ALT, the MRN complex closely associates with other recombination proteins involved in recombination-dependent telomere elongation. Interestingly, use of short-hairpin RNA to knockdown the Rad50 gene induced rapid telomere shortening that was significantly associated with the extent of gene knockdown and that occurred in a shorter timeframe than telomeric attrition resulting from inactivation of telomerase (46). Finally, compelling evidence implicating $\operatorname{Rad} 50$ in telomere rapid deletion comes from mice that are homozygous for a hypomorphic mutation in Rad50. These mice demonstrated a high frequency of fusions between chromosomes with shortened telomeres, correlating with the rapid loss of the originally elongated telomere (51). We propose that mutant RAD50 induces rapid telomere shortening through a combination of factors that may include interruption of telomerase action, deactivation of ALT mechanisms, and chromosomal rearrangements secondary to compromise of telomeric protection. Although our current study does not extend into this area, this mechanism represents a future direction for study, which we will be pursuing. Of note, cisplatin also degrades telomeres through the preferential platination of repeat guanine residues in human telomeres (43). Indeed, combination treatment with cisplatin and Ad-RAD50 caused significantly greater telomere shortening than either treatment alone. DNA damage induced by platination is likely to contribute to the observed telomeric shortening.

Experiments on human head and neck SCC tumor xenografts indicated that the induction of DNA DSB damage and telomere shortening by Ad-RAD50 produces a biological effect in vivo. Cisplatin alone did not demonstrate antitumor efficacy, as defined by the NCI, when used on tumors mirroring the resistance to cisplatin observed clinically. However, cisplatin-induced tumor volume reduction was dramatically enhanced by combination therapy with Ad-RAD50, which acted to overcome chemoresistance, resulting in a significant antitumor effect according to NCI criteria. This effect could be partially attributable to the observed increase in apoptosis. A key observation was that tumors treated with a combination of Ad-RAD50 and $3 \mathrm{mg} / \mathrm{kg}$ of cisplatin were, on average, over $30 \%$ smaller than tumors treated with cisplatin $5 \mathrm{mg} / \mathrm{kg}$ alone. This finding has important clinical implications, as a shift to lower dose cisplatin therapy could greatly reduce or eliminate treatmentlimiting toxicities that are common with higher doses of cisplatin given alone or in combination with radiation therapy.

In summary, the present study defines what we believe to be a novel approach to improve the efficacy of cisplatin-based chemotherapy for resistant tumors, which focuses on the interruption of MRN DNA repair function through targeted disruption of the RAD50 component. Cisplatin is the most effective antitumor drug for head and neck cancer patients and is the first-line treat- 
A

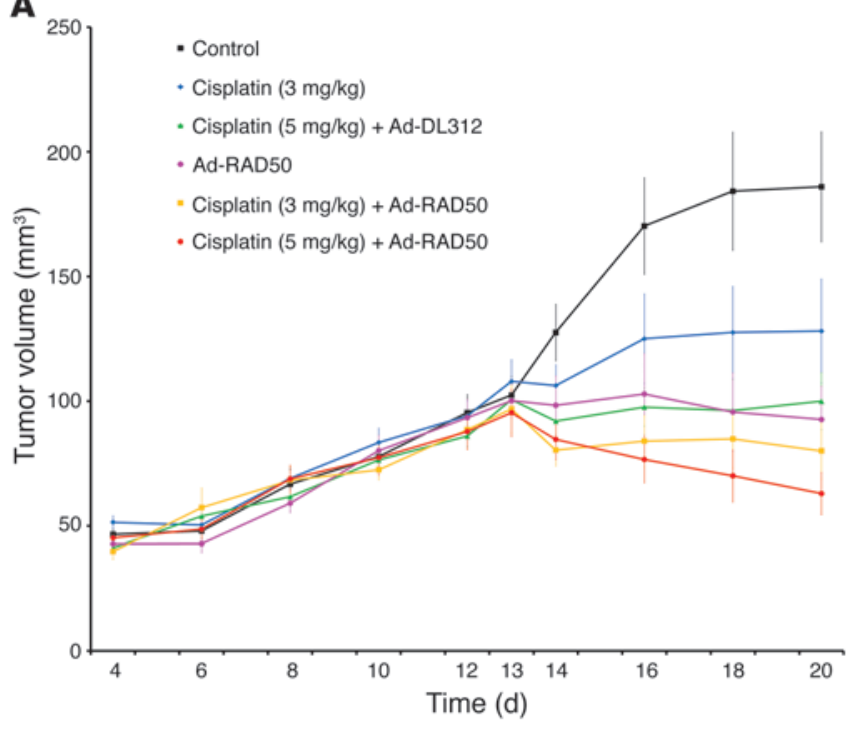

B

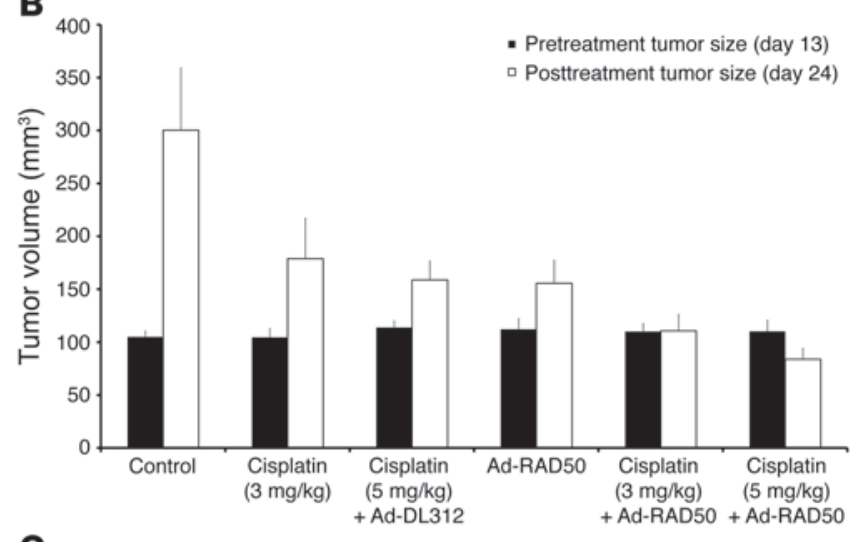

C

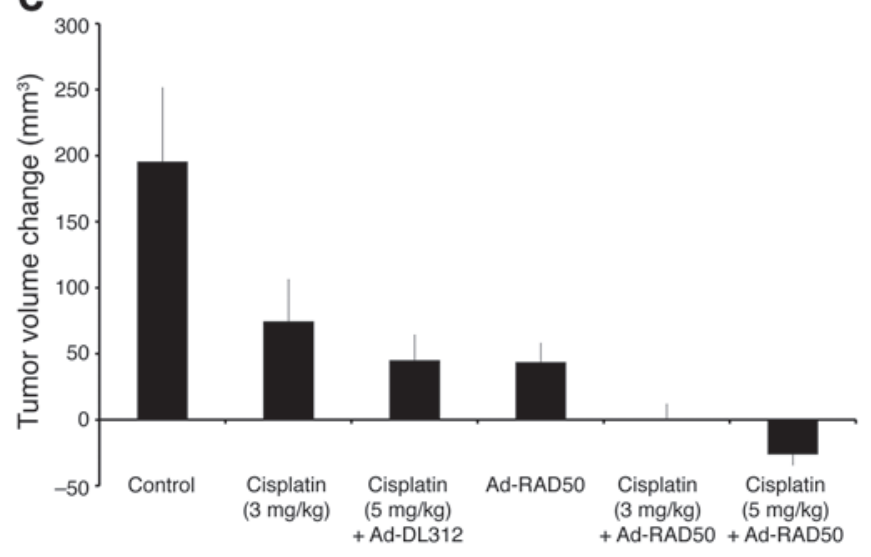

\section{Figure 7}

Ad-RAD50 chemosensitizes JHU012 human head and neck cancer to cisplatin in vivo. (A) Externally measured tumor volume \pm SEM from the time of initial tumor palpability on day 4, to treatment initiation on day 13, and through the treatment period to day 20. (B) Mean internally measured tumor volume \pm SEM immediately prior to treatment on day 13 and at the time of animal sacrifice on day 24. (C) Mean internally measured change in tumor volume \pm SEM. During the 11 day period between treatment initiation and animal sacrifice, a single administration of Ad-RAD50 combined with a single intraperitoneal dose of cisplatin, at either $3 \mathrm{mg} / \mathrm{kg}$ or $5 \mathrm{mg} / \mathrm{kg}$, was able to significantly enhance the therapeutic efficacy of cisplatin (3 mg/kg or $5 \mathrm{mg} / \mathrm{kg})$ monotherapy. For Ad-RAD50 with cisplatin $(3 \mathrm{mg} / \mathrm{kg}), P<0.001 \mathrm{vs}$. control; $P<0.05 \mathrm{vs}$. cisplatin $(3 \mathrm{mg} / \mathrm{kg})$ and cisplatin ( $5 \mathrm{mg} / \mathrm{kg}$ ) with DL312; and $P<0.05 \mathrm{vs.} \mathrm{Ad-RAD50} \mathrm{alone.} \mathrm{For} \mathrm{Ad-RAD50} \mathrm{with} \mathrm{cisplatin}(5 \mathrm{mg} / \mathrm{kg}), P<0.001 \mathrm{vs}$. control, cisplatin (3 $\mathrm{mg} / \mathrm{kg})$, cisplatin (5 mg/kg) with DL312, and Ad-RAD50 alone.

ment for many other cancers. By introducing the newly developed RAD50-disrupting vector, Ad-RAD50, and illustrating the beneficial effects of this therapeutic approach when combined with standard cisplatin chemotherapy, this study may lead to a new strategy for unraveling chemoresistance and could be translated to patient treatment with a potentially major impact on disease outcome.

\section{Methods}

Cell lines. The JHU012 and JHU029 human SCC cell lines were originally derived from human head and neck tumor explants, obtained from 2 patients with advanced head and neck SCC, during primary surgical resection of the tumor. Fully informed consent was obtained from these patients for the use of their tissues in research. These cell lines were generated at the Johns Hopkins University Head and Neck Biological Research Laboratories and have since been propagated and maintained in our laboratory at the University of Pennsylvania School of Medicine. Both cell lines have been well characterized $(36,40,52,53)$. Cell lines were screened for wild-type Mre11, Rad50, and Nbs1 expression as well as $R b$ and $p 53$ gene expression using Western blotting as previously described $(35,36,38,52,54)$. Cells were cloned from the original parental cell lines for use in all experiments. Cells were cultured in RPMI 1640 media, supplemented with $10 \%$ fetal bovine serum and $1 \%$ penicillin-streptomycin at $37^{\circ} \mathrm{C}$ in $5 \% \mathrm{CO}_{2}$.

Construction of the recombinant adenoviral vector. The AdEasy system (provided by T.C. He, Johns Hopkins Oncology Center, Baltimore, Maryland, USA) was used to construct the adenoviral vector (55). A plasmid containing a fragment of the RAD50 hook situated at the hinge domain of the wild-type RAD50 gene was cloned into a pAdTrack-CMV shuttle vector. The plasmid was linearized by PmeI digestion, cotransformed into E. coli BJ5183 cells with the pAdEasy- 1 adenoviral backbone plasmid, and then transfected into HEK293 cells to generate the recombinant adenovirus, Ad-RAD50. Ad-RAD50 was amplified and purified, and titers were determined using standard plaque assay. The control adenovirus, DL312 with E1a region deletion, was obtained from Tom Shenk, Princeton University, Princeton, New Jersey, USA.

MRN complex protein expression in SCC cells. The JHU012 and JHU029 cell lines were screened for wild-type MRE11, RAD50, and NBS1 expression using Western blot. Primary antibodies used were polyclonal rabbit anti-human MRE11 (Novus Biologicals), polyclonal rabbit anti-human RAD50 (Novus 
A

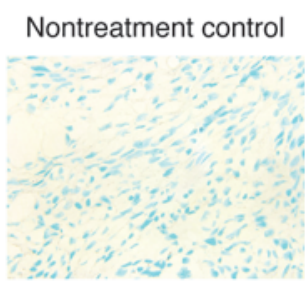

Cisplatin $(5 \mathrm{mg} / \mathrm{kg})$

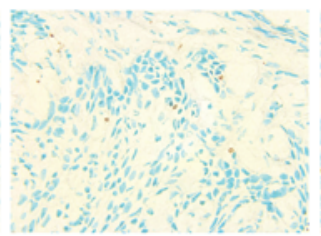

Cisplatin $(3 \mathrm{mg} / \mathrm{kg})$

Ad-RAD50

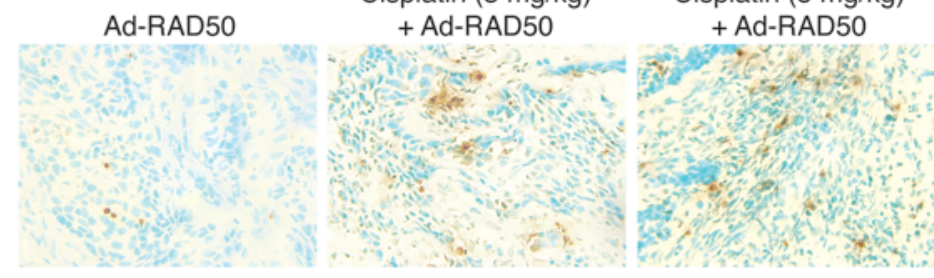

B

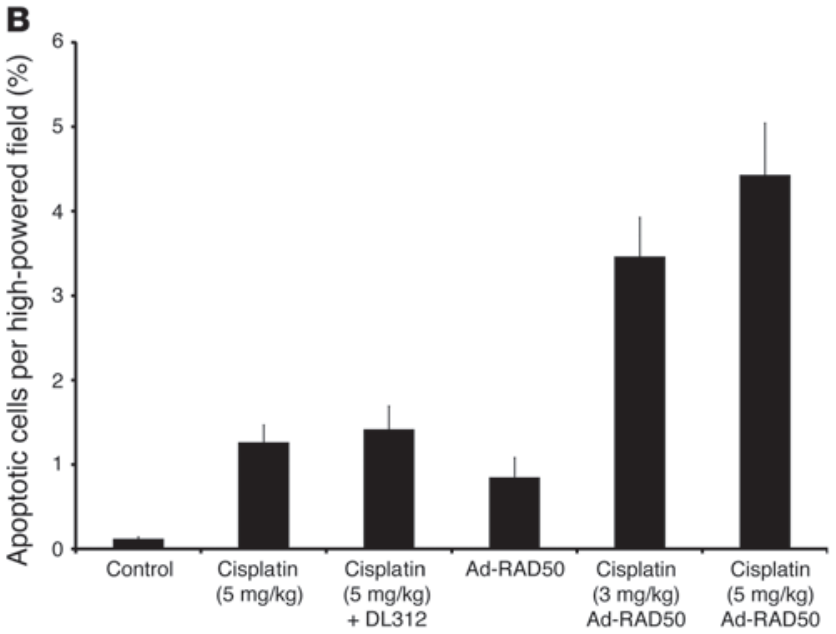

Figure 8

Quantification of apoptosis in human head and neck cancer tumors. (A) TUNEL staining was performed on tumor SCC samples after tumor harvest. A selection of 1 high-powered field (original magnification, $\times 40$ ) from each treatment group is shown. (B) Mean percentage apoptosis per high-powered field \pm SEM is shown. The combination of Ad-RAD50 and cisplatin enhanced induction of apoptosis relative to all other groups $(P<0.001)$. This is consistent with the tumor volume reduction seen in the combination therapy groups.

Biologicals), polyclonal rabbit anti-human NBS1 (Novus Biologicals), and polyclonal rabbit anti- $\beta$ actin (Novus Biologicals). Following infection of JHU012 cells with Ad-RAD50, expression of mutant RAD50 was confirmed using Western blot, as previously described (11). Recombinant adenovirus was introduced into JHU012 cell cultures at a MOI of 10 for 4 hours at $37^{\circ} \mathrm{C}$. The primary antibodies used were a polyclonal rabbit anti-human RAD50 (zinc hook domain) (Novus Biologicals), which recognizes both wild-type and mutant RAD50, and the same polyclonal rabbit anti-human antibodies targeted against MRE11 and NBS1 that were used in the preceding screening experiment. For all Western blot experiments, primary antibodies were used at a concentration of 1:1,000. WesternBreeze Chemiluminescent immunodetection system (Invitrogen) was used to observe protein bands. MagicMark Western Protein Standard (Invitrogen) was used for protein molecular weight estimation. Experiments were repeated in triplicate. Images of gels were captured using a Microtek scanner (Microtek). ImageJ version 1.38 software (http://rsb.info.nih.gov/ij/), an established image analysis program available in the public domain through the National Institutes of Health, was used to analyze and quantify the density of Western blot bands.
Coimmunoprecipitation of MRN proteins. Coimmunoprecipitation and immunoblotting experiments were conducted to verify the binding interactions between mutant RAD50 and the MRN complex. JHU012 cells were either untreated or infected with Ad-RAD50 at an MOI of 20, twenty-four hours after plating. Eight hours after infection, a subset of cells from the untreated and Ad-RAD50 infected group underwent treatment with cisplatin $(0.3 \mu \mathrm{g} / \mathrm{ml})$ overnight. Cell lysis buffer $(0.5 \%$ Nonidet P-40, $10 \mathrm{mM}$ sodium phosphate buffer at $\mathrm{pH} 7.6,20 \mathrm{mM}$ HEPES at pH 7.6, $150 \mathrm{mM} \mathrm{NaCl}, 10 \mathrm{mM}$ EDTA, 10\% glycerol, $10 \mathrm{mM} \mathrm{NaF}, 1 \mathrm{mM} \mathrm{Na}_{3} \mathrm{VO}_{4}$, and protease inhibitor cocktail) was added to the cells. Lysate was centrifuged at 13,000 $\mathrm{g}$ for 10 minutes at $4^{\circ} \mathrm{C}$. Supernatant was retrieved and protein content was quantified using the Bradford protein assay (Bio-Rad). Lysate preclearing was performed by rolling the lysate with protein A-Sepharose CL-4B beads (Pfizer-Pharmacia) at $4^{\circ} \mathrm{C}$ on a microcentrifuge tube roller apparatus. Cleared lysates were then incubated with mouse anti-hMre11 antibody (Novus Biologicals) at a concentration of 1:1,000 and placed on the roller for 1 hour at $4{ }^{\circ} \mathrm{C}$. Immune complexes were then precipitated by rolling the lysates with protein A-Sepharose CL-4B beads overnight. Immunoprecipitates bound to beads were washed 3 times with cell lysis buffer. Zinc chloride was added. After boiling in SDS sample buffer, immunoprecipitates were analyzed on $8 \%$ SDS-PAGE gel followed by transfer to $0.45-\mu \mathrm{m}$ PVDF membrane (Invitrogen). Nonspecific binding sites were blocked using $5 \%$ nonfat dried milk in $10 \mathrm{mM}$ Tris- $\mathrm{HCl}$ buffer at $\mathrm{pH} 7.5,125 \mathrm{mM} \mathrm{NaCl}$, and $0.1 \%$ Tween 20. Polyclonal rabbit anti-hRad50 (zinc hook region) was used as the primary antibody and incubated with the samples overnight at $4{ }^{\circ} \mathrm{C}$. Goat anti-rabbit secondary antibody conjugated to horseradish peroxidase was then applied to the membranes at a concentration of 1:5,000. PVDF membranes were rinsed and protein bands were visualized using the WesternBreeze Chemiluminescent immunodetection system (Invitrogen). SeeBlue Plus2 PreStained Standard (Invitrogen) was used to monitor electrophoresis progression and to evaluate transfer efficiency.

Visualization of MRE11/RAD50 binding interactions by confocal microscopy. JHU012 cells were plated at a concentration of $2 \times 10^{5}$ in $100-\mathrm{mm}$ tissue culture dishes and allowed to adhere overnight. Cells were left untreated or infected with Ad-RAD50 (MOI 20) for 4 hours at $37^{\circ} \mathrm{C}$. Adenoviral transgene induction was confirmed by visualizing GFP expression under fluorescence microscopy 12 hours after infection. Cells were transferred to 2 Lab-Tek II 8-well Chamber Slides (Thermo Fisher Scientific) at a concentration of $3 \times 10^{4}$ cells per well and allowed to adhere overnight. Cisplatin was then instilled at $0.3 \mu \mathrm{g} / \mathrm{ml}$. Each Lab-Tek II slide was configured as follows: control cells (wells 1 and 5), cisplatin-treated cells (wells 2 and 6), Ad-RAD50-infected cells (wells 3 and 7), and Ad-RAD50 with cisplatin-treated cells (wells 4 and 8). Wells 1-4 were treated with mouse monoclonal anti-human RAD50 (13B3) (Novus Biologicals) and goat polyclonal anti-human MRE11 (Santa Cruz Biotechnology) primary antibodies. Donkey anti-mouse Alexa Fluor 555 and donkey anti-goat Alexa Fluor 647 (Invitrogen) were used as secondary antibodies. Wells 5-8 were treated with mouse monoclonal anti-human RAD50 (13B3) and rabbit polyclonal anti-human RAD50 (zinc hook region) (Novus Biologicals) primary antibodies, followed by donkey antimouse Alexa Fluor 555 and donkey anti-rabbit Alexa Fluor 647 (Invitrogen) secondary antibodies. Wells $1-4$, on the second Lab-Tek II slide, served as controls and were not with any antibodies. Wells 5-8 were treated with only secondary antibodies. Slides were fixed using Prolong Gold Antifade 
(Invitrogen) mounting media. Cell nuclei were viewed with a Zeiss LSM 510 confocal microscope at $\times 63$ magnification. The 488 -nm laser line was used to excite GFP and to identify Ad-RAD50-infected cells. Alexa Fluor 555 and 647 secondary antibodies were excited using the $543-\mathrm{nm}$ and $633-\mathrm{nm}$ lasers, respectively. Images were captured using LSM 510 software (Zeiss) and analyzed for signal colocalization. This experiment was repeated in triplicate.

In vitro effects of Ad-RAD50 gene transfer on cell growth and survival. Three samples of $3 \times 10^{3} \log$ phase cells were plated in 96-well tissue culture plates. Cells were then incubated with Ad-RAD50 or DL312 (MOI 10) for 4 hours at $37^{\circ} \mathrm{C}$. Cisplatin (Bristol-Myers Squibb) was added to cultures 24 hours after viral infection. Cisplatin was instilled at a concentration of $0.3 \mu \mathrm{g} / \mathrm{ml}$ and $0.4 \mu \mathrm{g} / \mathrm{ml}$ into each JHU012 and JHU029 well, respectively. This cisplatin concentration represents the $\mathrm{IC}_{50}$ dose for each cell line. Cell growth over 6 days was determined by MTT assay.

Neutral comet assay. Neutral comet assay was used to quantify DNA DSBs as described previously $(11,39)$. JHU012 and JHU029 cancer cells that were not treated and cells treated with the replication defective DL312 empty virus served as controls. The other 4 treatment groups were cisplatin alone (JHU012, $0.3 \mu \mathrm{g} / \mathrm{ml}$; JHU029, 0.4 mg/ml), Ad-RAD50 alone, DL312 with cisplatin, and Ad-RAD50 with cisplatin. Tumor cells from the JHU012 and JHU029 cell lines were grown as a monolayer in 24-well tissue culture plates. Cells were infected with either Ad-RAD50 or DL312 (MOI 10) over 4 hours at $37^{\circ} \mathrm{C}$. Twenty-four hours after the time of infection, a subset of the cells were treated with cisplatin for 2 hours at $37^{\circ} \mathrm{C}$. The neutral comet assay was performed at 24,48 , and 72 hours. Slides were viewed at $\times 40$ magnification using a Nikon microscope, and images of 30-70 randomly selected cells per sample group were acquired using a SPOT-2 CCD Camera (Diagnostic Instruments). Comet measurements and quantitative analysis were performed using Scion Imaging software (56). The measured tail moment is directly proportional to the quantity of DNA DSBs. MTM was derived from 30-70 tumor cells per sample group.

Telomere length analysis. JHU012 cancer cells were used for this analysis. Treatment groups were identical to those used for the neutral comet assay as above, and adenoviral vectors were introduced at a MOI of 10. Cisplatin was used at the $\mathrm{IC}_{50}$ dose for the JHU012 cell line $(0.3 \mu \mathrm{g} / \mathrm{ml})$. Cells were incubated for 96 hours (40). Culture medium was supplemented with $10 \mu \mathrm{g} / \mathrm{ml} \mathrm{Colcemid} \mathrm{(Gibco)} \mathrm{for} 2$ hours. Cells were treated with $60 \mathrm{mM}$ $\mathrm{KCl}$, pelleted by centrifugation, and resuspended in fixative (3:1 methanol/ acetic acid). The suspensions were applied to slides and stored in $20^{\circ} \mathrm{C}$ ethanol for 48-72 hours. In situ hybridization was performed using the Telomere PNA FISH Kit (Dako) according to manufacturer's instructions. Cell staining was performed with $2 \times 5 \mu \mathrm{l}$ Vectashield anti-fade solution (Vector Laboratories), containing $0.2 \mu \mathrm{g} / \mathrm{ml}$ DAPI as a counterstain. Images were captured, and 4 randomly selected high-powered fields on each slide were analyzed. Telomere-specific staining intensity was quantified using IPLab image analysis software (BD Biosciences).

Animals. Animal experiments were conducted in 8- to 10-week-old athy$\mathrm{mic} \mathrm{BALB} / \mathrm{c}$ nu/nu nude mice. The use of animals in this study was reviewed and approved by the University of Pennsylvania School of Medicine Institutional Animal Care and Use Committee.

Ad-RAD50 treatment of human head and neck cancer xenografts. Sixty nude mice were randomized into 6 groups of 10 mice each. Mice were anesthe- tized with $8 \mathrm{mg}$ tribromoethanol via intraperitoneal injection. Depth of anesthesia was determined by toe pinch. The right dorsal flank of each mouse was injected subcutaneously with $1 \times 10^{7} \mathrm{JHU} 012$ cells. After the establishment of palpable tumors, mouse body weight and external tumor volume were determined regularly. External tumor diameter was measured using digital calipers, and tumor volume was calculated using the formula $A^{2} \times B \times 0.536$, where $A$ represents the smallest diameter and $B$ equals the largest diameter. Mice were reanesthetized 13 days after tumor injection, when the average externally measured tumor volume was $100 \mathrm{~mm}^{3}$. Tumors were exposed by raising a skin flap using precise surgical dissection, taking care not to disturb the tumor or its blood supply. The exposed tumors were measured in 3 dimensions (maximal length $\times$ maximal width $\times$ maximal height), using calipers to accurately assess tumor volume as previously described (36). The intratumoral dose of Ad-RAD50 and DL312 was $8.5 \times 10^{8} \mathrm{PFUs}$ in $50 \mu \mathrm{l}$ volume. The 6 intervention groups were intratumoral saline, intraperitoneal cisplatin ( $3 \mathrm{mg} / \mathrm{kg})$, intratumoral DL312 and intraperitoneal cisplatin $(5 \mathrm{mg} / \mathrm{kg})$, intratumoral Ad-RAD50, intratumoral Ad-RAD50 and intraperitoneal cisplatin $(3 \mathrm{mg} / \mathrm{kg})$, and intratumoral Ad-RAD50 with intraperitoneal cisplatin ( $5 \mathrm{mg} / \mathrm{kg})$. Skin incisions were closed with 4-0 nylon suture. The internal tumor volume was remeasured 11 days after treatment.

Quantification of apoptosis in human head and neck SCC tumors. Tumors were harvested from each mouse after size measurement. Harvested tumors were bisected and snap frozen in liquid nitrogen, embedded in OCT matrix, sectioned into $7-\mu \mathrm{M}$ thick samples, and mounted on super-frosted glass slides. TUNEL apoptosis staining using an ApopTag Plus kit (Chemicon) was performed according to manufacturer's instructions. After staining, 5 tumor specimens were randomly selected from each treatment group. Ten $\times 40$ high-powered fields from each specimen were randomly selected. Images were acquired using the imaging system described above. IPLab software was used to determine the percentage of each high-powered field consisting of DAB-stained apoptotic cells.

Statistics. Statistical analysis was performed with StatMost (Dataxiom Software). The 2-tailed Student's $t$ test for independent samples was used for the analysis of all data, with the exception of the in vivo tumor volume experiment, which was analyzed using the Mann-Whitney $U$ test. $P$ values of less than 0.05 were considered to be statistically significant.

\section{Acknowledgments}

This work received grant support from the Flight Attendant Medical Research Institute and from the National Institute of Dental and Craniofacial Research (5R01 DE014562).

Received for publication September 4, 2007, and accepted in revised form March 19, 2009.

Address correspondence to: Daqing Li, Department of Otorhinolaryngology - Head and Neck Surgery, University of Pennsylvania School of Medicine, 415 Curie Boulevard, Clinical Research Building (CRB) Room 145, Philadelphia, Pennsylvania 19104, USA. Phone: (215) 615-0854; Fax: (215) 573-1934; E-mail: lidaqing@ mail.med.upenn.edu.
1. Kelland, L. 2007. The resurgence of platinum-based cancer chemotherapy. Nat. Rev. Cancer. 7:573-584.

2. Chu, G. 1994. Cellular responses to cisplatin. The roles of DNA-binding proteins and DNA repair. J. Biol. Chem. 269:787-790.

3. Perez, R.P. 1998. Cellular and molecular determinants of cisplatin resistance. Eur. J. Cancer. 34:1535-1542.

4. Rodenburg, C.J., and Cleton, F.J. 1984. Chemother- apy in advanced ovarian cancer. J. Cancer Res. Clin. Oncol. 107:99-105.

5. Jacobs, C., et al. 1992. A phase III randomized study comparing cisplatin and fluorouracil as single agents and in combination for advanced squamous cell carcinoma of the head and neck. J. Clin. Oncol. 10:257-263.

6. Vokes, E.E., and Athanasiadis, I. 1996. Chemotherapy of squamous cell carcinoma of head and neck: the future is now. Ann. Oncol. 7:15-29.

7. Drummond, J.T., Anthoney, A., Brown, R., and Modrich, P. 1996. Cisplatin and adriamycin resistance are associated with MutLalpha and mismatch repair deficiency in an ovarian tumor cell line. J. Biol. Chem. 271:19645-19648.

8. Fink, D., et al. 1996. The role of DNA mismatch repair in platinum drug resistance. Cancer Res. 56:4881-4886 
9. Kartalou, M., and Essigmann,J.M. 2001. Mechanisms of resistance to cisplatin. Mutat. Res. 478:23-43.

10. O'Malley, B.W., Jr., Li, D., Carney, J., Rhee, J., and Suntharalingam, M. 2003. Molecular disruption of the MRN(95) complex induces radiation sensitivity in head and neck cancer. Laryngoscope. 113:1588-1594.

11. Tran, H.M., et al. 2004. Mutant Nbs1 enhances cisplatin-induced DNA damage and cytotoxicity in head and neck cancer. Otolaryngol. Head Neck Surg. 131:477-484.

12. Hopfner, K.P., Putnam, C.D., and Tainer, J.A. 2002. DNA double-strand break repair from head to tail. Curr. Opin. Struct. Biol. 12:115-122.

13. Stavridi, E.S., and Halazonetis, T.D. 2005. Nbs1 moving up in the world. Nat. Cell Biol. 7:648-650.

14. Maser, R.S., Monsen, K.J., Nelms, B.E., and Petrini, J.H. 1997. hMre11 and hRad50 nuclear foci are induced during the normal cellular response to DNA double-strand breaks. Mol. Cell. Biol. 17:6087-6096.

15. Zhang, Y., Zhou, J., and Lim, C.U. 2006. The role of NBS1 in DNA double strand break repair, telomere stability, and cell cycle checkpoint control. Cell Res. 16:45-54.

16. Assenmacher, N., and Hopfner, K.P. 2004. MRE11/ RAD50/NBS1: complex activities. Chromosoma. 113:157-166.

17. Bhaskara, V., et al. 2007. Rad50 adenylate kinase activity regulates DNA tethering by Mre11/Rad50 complexes. Mol. Cell. 25:647-661.

18. Dolganov, G.M., et al. 1996. Human Rad50 is physically associated with human Mre11: identification of a conserved multiprotein complex implicated in recombinational DNA repair. Mol. Cell. Biol. 16:4832-4841.

19. Hopfner, K.P., et al. 2000. Structural biology of Rad50 ATPase: ATP-driven conformational control in DNA double-strand break repair and the ABCATPase superfamily. Cell. 101:789-800.

20. Anderson, D.E., Trujillo, K.M., Sung, P., and Erickson, H.P. 2001. Structure of the Rad50 x Mre11 DNA repair complex from Saccharomyces cerevisiae by electron microscopy. J. Biol. Chem. 276:37027-37033.

21. Hopfner, K.P., et al. 2001. Structural biochemistry and interaction architecture of the DNA doublestrand break repair Mre11 nuclease and Rad50ATPase. Cell. 105:473-485.

22. Paull, T.T., and Gellert, M. 1999. Nbs1 potentiates ATP-driven DNA unwinding and endonuclease cleavage by the Mre11/Rad50 complex. Genes Dev. 13:1276-1288.

23. Lee, J.H., and Paull, T.T. 2005. ATM activation by DNA double-strand breaks through the Mre11Rad50-Nbs1 complex. Science. 308:551-554.

24. Wiltzius, J.J., Hohl, M., Fleming, J.C., and Petrini,
J.H. 2005. The Rad50 hook domain is a critical determinant of Mre11 complex functions. Nat. Struct. Mol. Biol. 12:403-407.

25. Moreno-Herrero, F., et al. 2005. Mesoscale conformational changes in the DNA-repair complex Rad50/Mre11/Nbs1 upon binding DNA. Nature. 437:440-443.

26. Hopfner, K.P., et al. 2002. The Rad50 zinc-hook is a structure joining Mre11 complexes in DNA recombination and repair. Nature. 418:562-566.

27. Zhong, H., Bryson, A., Eckersdorff, M., and Ferguson, D.O. 2005. Rad50 depletion impacts upon ATR-dependent DNA damage responses. Hum. Mol. Genet. 14:2685-2693.

28. Zhu, X.D., Kuster, B., Mann, M., Petrini, J.H., and de, L.T. 2000. Cell-cycle-regulated association of RAD50/MRE11/NBS1 with TRF2 and human telomeres. Nat. Genet. 25:347-352.

29. Chai, W., Sfeir, A.J., Hoshiyama, H., Shay, J.W., and Wright, W.E. 2006. The involvement of the Mre11/ Rad50/Nbs1 complex in the generation of G-overhangs at human telomeres. EMBO Rep. 7:225-230.

30. Wu, Y., Xiao, S., and Zhu, X.D. 2007. MRE11RAD50-NBS1 and ATM function as co-mediators of TRF1 in telomere length control. Nat. Struct. Mol. Biol. 14:832-840.

31. Zhu, X.D., Kuster, B., Mann, M., Petrini, J.H., and de Lange, T. 2000. Cell-cycle-regulated association of RAD50/MRE11/NBS1 with TRF2 and human telomeres. Nat. Genet. 25:347-352.

32. Tauchi, H., Matsuura, S., Kobayashi, J., Sakamoto, S., and Komatsu, K. 2002. Nijmegen breakage syndrome gene, NBS1, and molecular links to factors for genome stability. Oncogene. 21:8967-8980.

33. Fukuda, T., et al. 2001. Alterations of the doublestrand break repair gene MRE11 in cancer. Cancer Res. 61:23-26.

34. Heikkinen, K., et al. 2006. RAD50 and NBS1 are breast cancer susceptibility genes associated with genomic instability. Carcinogenesis. 27:1593-1599.

35. Richtsmeier, W.J., and Carey, T.E. 1987. Rationalized nomenclature for head and neck carcinomas. Arch. Otolaryngol. Head Neck Surg. 113:1339-1340.

36. Rocco, J.W., et al. 1998. p16INK4A adenovirus-mediated gene therapy for human head and neck squamous cell cancer. Clin. Cancer Res. 4:1697-1704.

37. Rocco, J.W., Leong, C.O., Kuperwasser, N., DeYoung M.P., and Ellisen, L.W. 2006. p63 mediates survival in squamous cell carcinoma by suppression of $\mathrm{p} 73$ dependent apoptosis. Cancer Cell. 9:45-56.

38. Scher, R.L., Koch, W.M., and Richtsmeier, W.J. 1993. Induction of the intercellular adhesion molecule (ICAM-1) on squamous cell carcinoma by interferon gamma. Arch. Otolaryngol. Head Neck Surg. 119:432-438.

39. Olive, P.L., and Banath, J.P. 2006. The comet assay: a method to measure DNA damage in individual cells. Nat. Protoc. 1:23-29.

40. Li, D., et al. 2002. The role of adenovirus-mediated retinoblastoma 94 in the treatment of head and neck cancer. Cancer Res. 62:4637-4644.

41. Ames, B.N., and Gold, L.S. 1991. Endogenous mutagens and the causes of aging and cancer. Mutat. Res. 250:3-16.

42. Le, S., Moore, J.K., Haber, J.E., and Greider, C.W. 1999. RAD50 and RAD51 define two pathways that collaborate to maintain telomeres in the absence of telomerase. Genetics. 152:143-152.

43. Ishibashi, T., and Lippard, S.J. 1998. Telomere loss in cells treated with cisplatin. Proc. Natl. Acad. Sci. U.S. A. 95:4219-4223.

44. Bissery, M.C., Guenard, D., Gueritte-Voegelein, F., and Lavelle, F. 1991. Experimental antitumor activity of taxotere (RP 56976, NSC 628503), a taxol analogue. Cancer Res. 51:4845-4852.

45. Sorenson, C.M., and Eastman, A. 1988. Mechanism of cis-diamminedichloroplatinum(II)-induced cytotoxicity: role of G2 arrest and DNA doublestrand breaks. Cancer Res. 48:4484-4488.

46. Zhong, Z.H., et al. 2007. Disruption of telomere maintenance by depletion of the MRE11/RAD50/ NBS1 complex in cells that use alternative lengthening of telomeres. J. Biol. Chem. 282:29314-29322.

47. Carney, J.P., et al. 1998. The hMre11/hRad50 protein complex and Nijmegen breakage syndrome: linkage of double-strand break repair to the cellular DNA damage response. Cell. 93:477-486.

48. Mao, L., et al. 1996. Telomerase activity in head and neck squamous cell carcinoma and adjacent tissues. Cancer Res. 56:5600-5604.

49. Murnane, J.P., Sabatier, L., Marder, B.A., and Morgan, W.F. 1994. Telomere dynamics in an immortal human cell line. EMBOJ. 13:4953-4962.

50. Lustig, A.J. 2003. Clues to catastrophic telomere loss in mammals from yeast telomere rapid deletion. Nat. Rev. Genet. 4:916-923.

51. Bender, C.F., et al. 2002. Cancer predisposition and hematopoietic failure in Rad50(S/S) mice. Genes Dev. 16:2237-2251.

52. Liggett, W.H., Jr., et al. 1996. p16 and p16 beta are potent growth suppressors of head and neck squamous carcinoma cells in vitro. Cancer Res. 56:4119-4123.

53. Cannon, T., et al. 2007. Comparison of animal models for head and neck cancer cachexia. Laryngoscope. 117:2152-2158.

54. Mao, L., et al. 1995. A novel p16INK4A transcript. Cancer Res. 55:2995-2997.

55. He, T.C., et al. 1998. A simplified system for generating recombinant adenoviruses. Proc. Natl. Acad. Sci. U. S. A. 95:2509-2514.

56. Helma, C., and Uhl, M. 2000. A public domain image-analysis program for the single-cell gel-electrophoresis (comet) assay. Mutat. Res. 466:9-15. 